\title{
Changes in somatosensory evoked potentials following an experimental focal ischaemic lesion in thalamus
}

\author{
A. Ladds *, N.M. Branston *, J. Vajda **, J.E. McGillicuddy *** and L. Symon * \\ * Gough-Cooper Department of Neurological Surgery, Institute of Neurology, Queen Square, London WC1 (U.K.), ** National Institute \\ of Neurosurgery, Amerikai 57, Budapest 1145 (Hungary), and *** Section of Neurosurgery, The University of Michigan, Ann Arbor, \\ MI 48109 (U.S.A.)
}

(Accepted for publication: 14 June 1987)

\begin{abstract}
Summary Experiments have been performed to produce localized thalamic ischaemia in baboons anaesthetised with alphachloralose. Somatosensory evoked potentials to median nerve stimulation were recorded in the medial lemniscus, VPL of thalamus and the primary somatosensory cortex. Local blood flow was also recorded by the hydrogen clearance technique in these regions. The early potential recorded in thalamus has been shown to be generated from 3 sources: (i) a positivity generated outside the VPL, (ii) local wavelets, most likely from synaptic activity close to the recording electrode, and (iii) a local overall negativity. The first of these potentials alone remains after thalamic ischaemia. It arises below the level of the thalamus, being very likely generated by the afferent volley in the medial lemniscus, and is seen in the surface-recorded response as the early component P8 (corresponding to P15 in the human).
\end{abstract}

Key words: Somatosensory evoked potentials; Thalamus; Ischaemia

The scalp-recorded somatosensory evoked potential (SEP) elicited by stimulation of the median nerve has provided a valuable tool for the clinician in assessing patients neurologically (e.g., Noël and Desmedt 1975). However, there is still uncertainty about the sources within the CNS responsible for generating the surface-recorded potentials. The first dominant potential obtained using a cephalic reference is known as P15 by some investigators and P14 by others. It has a diffuse distribution over the scalp (Rosner et al. 1963; Broughton 1969; Cracco 1972) and has been found by some investigators to persist in patients with lesions in the thalamus (Nakanishi et al. 1978, 1983; Crespi et al. 1982; Hammond et al. 1982; Suzuki and Mayanagi 1984), but by others

Correspondence to: Prof. L. Symon, Gough-Cooper Dept. of Neurological Surgery, Institute of Neurology, Queen Square, London WC1N 3BG (U.K.). to be lost (Cracco and Cracco 1976; Allison and Hume 1981; Stöhr et al. 1983). Some believe that P15 is the inversion of N14 as seen over the cervical spines (Cracco 1972; Jones 1977; Abbruzzese et al. 1978). Desmedt (1984) has shown from consideration of timing that the onset of P15 corresponds to the earliest activation of lemniscal axons at the cuneate nucleus and its peak to the time of arrival of the volley at the ventralis posterior lateralis (VPL) via the fastest lemniscal axons.

In previous work (Branston et al. 1984), the wave forms of SEPs recorded from the anaesthetised baboon have been compared with those obtained from the human when the contralateral median nerve is stimulated in each case. The first cortical negativity $\mathrm{N} 20$ recorded at the scalp in man corresponds to N10 in the baboon, while the scalp-recorded P15 in man appears in the baboon as a small but distinct positive peak with an average latency of about $8 \mathrm{msec}$ which we have, therefore, designated as P8. 
We have also previously shown a differential sensitivity between different levels of the neuraxis in respect of the maintenance of the SEP with decreased local blood flow (Branston et al. 1984). The threshold of flow at which the SEP fails is $15-20 \mathrm{ml} / 100 \mathrm{~g} / \mathrm{min}$ in the cerebral cortex, $10-15$ $\mathrm{ml} / 100 \mathrm{~g} / \mathrm{min}$ in the thalamus, and less than 10 $\mathrm{ml} / 100 \mathrm{~g} / \mathrm{min}$ in the medial lemniscus. In the present study, a knowledge of these thresholds has enabled us to interpret the changes observed in the SEPs recorded at the cortical surface and in the thalamus following the production of focal thalamic ischaemia and to obtain further information about the site of origin of P8.

\section{Methods}

Data were obtained from 9 baboons (Papio cynocephalus) in the weight range $7-12 \mathrm{~kg}$. The experimental protocol and the methods used to prepare the animals for surgery and for recording of electrophysiological and blood flow data have been fully described previously (Branston et al. 1984; Vajda et al. 1985). To summarise: the animals were anaesthetised with alpha-chloralose $(60 \mathrm{mg} / \mathrm{kg})$, immobilised with gallamine triethiodide (1 $\mathrm{mg} / \mathrm{kg}$, repeated as necessary) and maintained normocapnic by pump ventilation at appropriate stroke volume. Groups of electrodes $(0.8 \mathrm{~mm}$ diam. insulated stainless steel tubes, tipped with a $1 \mathrm{~mm}$ length of $0.25 \mathrm{~mm}$ diam. bared platinum wire) were placed stereotactically in and around the VPL for recording responses to contralateral median nerve stimulation and for recording local blood flow by the method of hydrogen clearance. Additional electrodes were placed in the medial lemniscus, over the postcentral gyrus (either epidurally or on the pia), and epidurally at C2. Flow (except at the neck) was measured concurrently with the SEP in these regions. Both the stimulating and recording electrode arrays were duplicated bilaterally.

SEPs were recorded using a stimulus rate of 1 $\mathrm{Hz}$, an amplifier bandpass of $1-6.4 \mathrm{kHz}$, with a gain of 10,000 , and a $25 \mathrm{msec}$ sweep time (256 sampled points). Averages of 256 sweeps were displayed and stored on disc for analysis with the
PDP-11 based system. Data were smoothed (once) using a 5-point, zero phase shift digital filter. All SEPs described below were recorded with the reference electrode at Fpz. In view of the clear demonstration by Desmedt and colleagues that in man the earliest scalp-recorded far-field components are essentially unmasked when a non-cephalic reference is used, it is interesting that in the baboon we have found that the use of a reference placed on the hand contralateral to the stimulus, instead of at Fpz, does not appreciably clarify these early components. This effect, which justifies our present choice of the Fpz reference site, has been illustrated and discussed elsewhere (Ladds et al. 1984) and may be accounted for by differences in neuroanatomical structure between species or in electrode geometry.

After the electrodes were positioned and fixed to the skull with dental cement, control measurements of SEPs and local flow were made. Then, via a trans-orbital approach, the major arteries supplying the thalamus on one side were identified and dissected free (Vajda et al. 1985). These included the thalamo-geniculate arteries from segment P2 of the posterior cerebral artery, the thalamo-perforators from $\mathrm{P} 1$, the posterior communicating and the posterior choroidal arteries. The vessels were then coagulated sequentially, with moderate controlled stepwise reductions of blood pressure by withdrawal of blood, as necessary, until on-line measurements of flow indicated that the VPL was unilaterally and densely ischaemic. This condition was verified subsequently by perfusion-fixation of the brain via the ascending aorta with a final injection of $3 \%$ gelatin in carbon black in order to outline the arterial tree and allow gross examination of brain sections (Vajda et al. 1985). After removal of the depth electrodes, frozen sections of the brain were made in which the positions of the electrode tips were clearly distinguishable in relation to surrounding structures; no special electrode marking technique was required.

\section{Results}

Fig. 1 illustrates the wave forms obtained in the normally perfused brain with serial recordings 


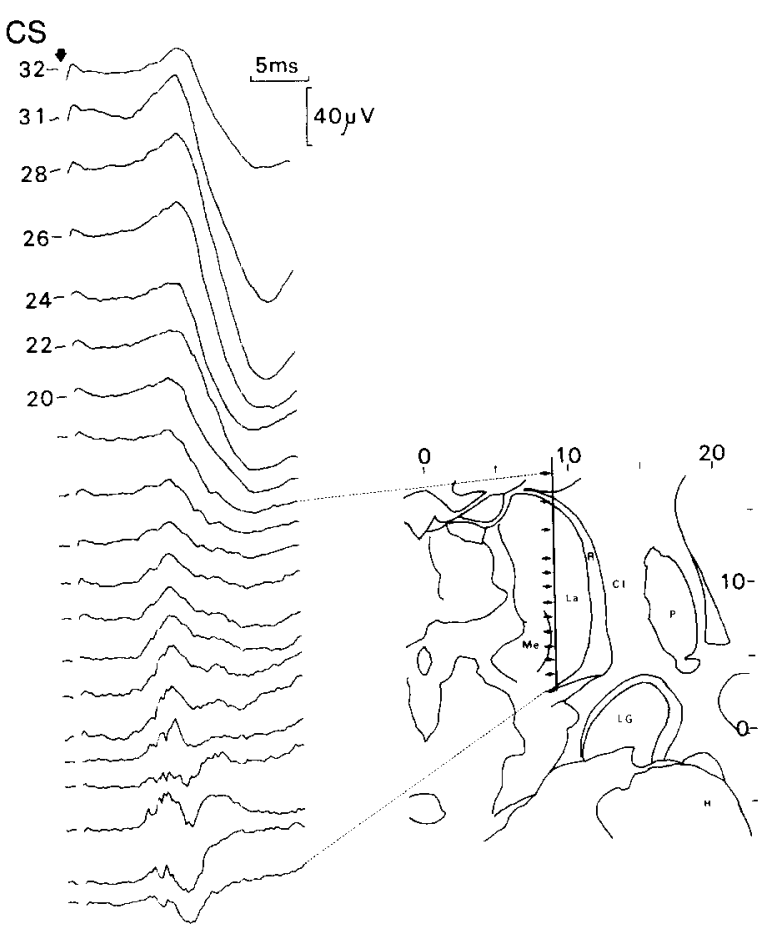

Fig. 1. Averaged evoked responses recorded during implantation of an electrode into the VPL. The numbers on the left are the vertical distance in $\mathrm{mm}$ above stereotactic zero. The top response was recorded at the cortical surface (CS). The responses between the dotted lines were recorded at sites indicated by arrows on the electrode tract in the stereotactic map of the VPL area. $\mathrm{La}=$ ventralis posterior lateralis; $\mathrm{R}=$ reticularis; $\mathbf{M e}=$ ventralis posterior medialis; $\mathrm{CI}=$ capsula interna; $\mathbf{P}=$ putamen; $\mathrm{Lg}=$ corporis geniculati; $\mathrm{H}=$ hippocampus. Reproduced by permission from Ladds et al. 1984.

made in a previous series (Ladds et al. 1984) during the stereotactic placement of an electrode in the VPL starting from the cortical surface, 32 $\mathrm{mm}$ above the stereotactic zero plane. At the surface, a positive potential of about $10 \mathrm{msec}$ latency is seen, followed by a negative potential at about $15 \mathrm{msec}$. As the electrode approaches the thalamus, the negativity disappears and the peak of the positive potential occurs earlier. In the VPL, the positive wave is more complex and includes small superimposed wavelets with a period of about 0.9 msec.

The changes in the thalamic response to ischaemia in the present series are illustrated in Fig. 2. In this animal, 6 thalamic electrodes were implanted in positions indicated on the stereotactic representations. The corresponding cortical recordings are also shown. Control recordings, indicated by (a), show a marked variation in amplitude and morphology of the VPL response and some wave forms, particularly from electrode 3, are very complex. However, in contrast to the diversity of wave form in the control phase, responses in the phase of dense ischaemia (b) were similar to each other, the main differences being in amplitude. The greatest amplitude was seen at the most lateral thalamic electrodes ( 3 and 6), with that at the most posterior and lateral (3) being largest. The early neck SEP components and the blood flow in the brain-stem were not significantly changed in ischaemia.

With the onset of ischaemia in these experiments, 3 observations were typically made. First, the small oscillatory wavelets seen in the VPL response disappeared; second, the amplitude of the VPL response was enhanced, the degree of enhancement depending on several factors (discussed below); and third, the cortical N10 and P15 components were abolished, leaving a residual surface potential with similar latency characteristics to the VPL response but with smaller amplitude. Since the local cortical flow was well above the threshold for loss of the cortical SEP (Branston et al. 1984), the abolition of cortical components must have resulted from loss of cortical input from the VPL itself. The blood flow in thalamus was below threshold and that in the brain-stem above threshold (Branston et al. 1984); a prerequisite for including data from any experiment in the present series was that the flow in cortex and brain-stem was in both above threshold, so that any changes in the evoked response would then be attributable to changes in thalamic activity.

Table I shows the average flow both in the control phase and after production of right thalamic ischaemia, in all recording areas. Flows in the right VPL and right cortex were significantly reduced with ischaemia. The flow in cortex, however, was still well above the threshold of 18 $\mathrm{ml} / 100 \mathrm{~g} / \mathrm{min}$ (Branston et al. 1984). Flows and SEPs on the left side were unaffected by right thalamic ischaemia. 

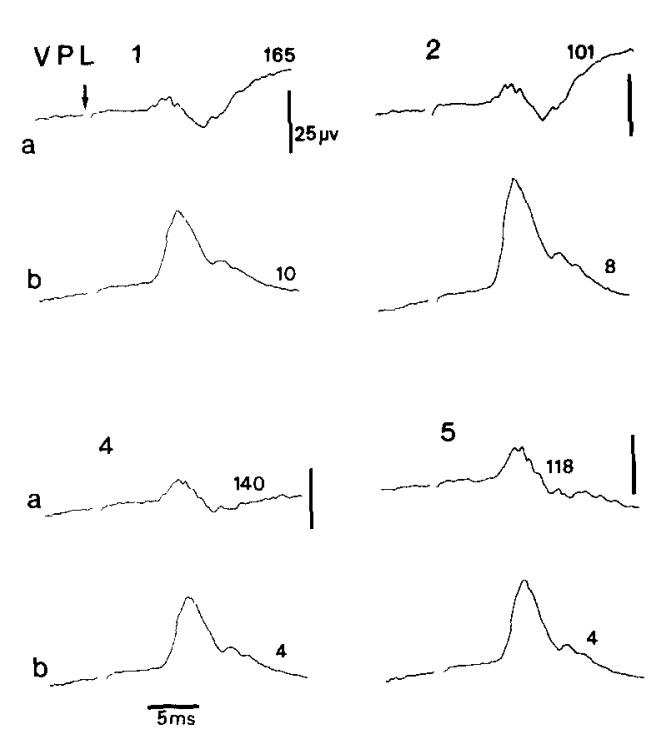
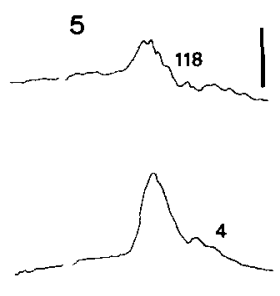
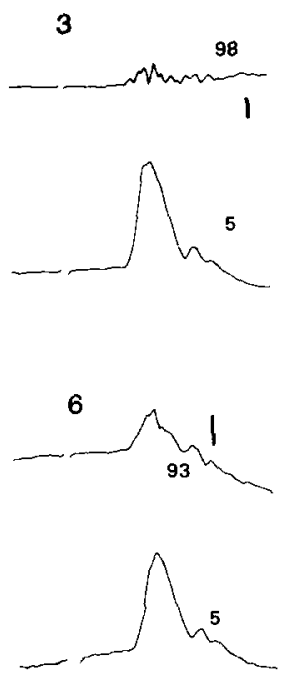

CORTEX

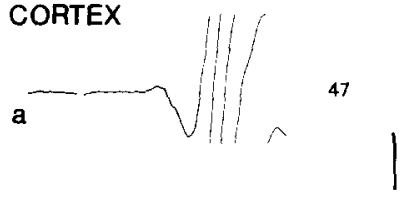

b

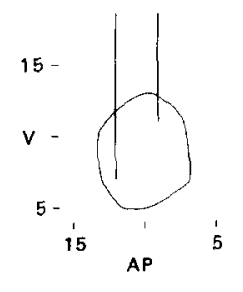

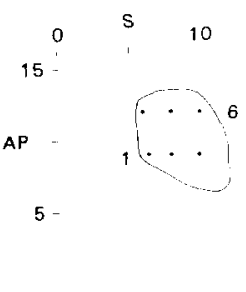

Fig. 2. Recordings within the VPL from 6 electrodes whose stereotactic positions are indicated in their anterior/posterior (AP), sagittal (S) and vertical (V) relationships in the bottom right of the diagram, both before (a) and after (b) production of thalamic ischaemia, in one animal. The corresponding changes in the cortical response are also shown. The blood flows (in units of ml/100 $\mathrm{g} / \mathrm{min}$ ) recorded at each site are also indicated.

Table II gives the average changes in the amplitude of the right thalamic response before and after ischaemia, for each experiment. There was a significant increase in amplitude of the VPL response with ischaemia; the standard deviations are high because of the large variation in amplitude increase recorded by different electrodes, as discussed below.

In 2 experiments, we positioned additional electrodes in front of and behind the VPL to measure the variation in size of the residual positive potential with position after ischaemia, in an attempt to

\section{TABLE I}

Blood flow ( $\mathrm{ml} / 100 \mathrm{~g} / \mathrm{min}$ ) recorded during the control phase and at the termination of the experiment in each area.

\begin{tabular}{llll}
\hline & Control & Final & \\
\hline RML & $62.4 \pm 21.1$ & $52.9 \pm 30.2$ & \\
RVPL & $79.8 \pm 14.4$ & $18.3 \pm 14.7$ & $P<0.001$ \\
R postcentral & $45.6 \pm 7.2$ & $36.1 \pm 3.8$ & $P<0.025$ \\
LML & $64.6 \pm 15.1$ & $60.1 \pm 33.3$ & \\
LVPL & $95.2 \pm 17.2$ & $95.6 \pm 31.8$ & \\
L postcentral & $44.4 \pm 6.7$ & $47.2 \pm 9.4$ & \\
\hline
\end{tabular}

identify the source of this potential. Fig. 3 shows the positioning of 10 electrodes in 1 animal, and Table III gives the corresponding amplitudes of the residual potential for each electrode. In this experiment, the electrodes surrounding the thalamus recorded only small positive potentials compared to those recorded within the VPL. Within the nucleus, there was a marked variation in size

\section{TABLE II}

Amplitude of the VPL potential $(\mu \mathrm{V})$ during the control phase and at the termination of each experiment. The difference between the mean values is significant at the $P=0.001$ level (paired $t$ test).

\begin{tabular}{lll}
\hline Exp. & Control & Final \\
\hline 1 & $16.76 \pm 12.56$ & $49.47 \pm 25.83$ \\
2 & $36.14 \pm 15.40$ & $41.05 \pm 9.06$ \\
3 & $12.62 \pm 7.58$ & $22.87 \pm 6.99$ \\
4 & $11.79 \pm 4.80$ & $14.57 \pm 6.99$ \\
5 & $39.57 \pm 21.32$ & $35.90 \pm 13.21$ \\
6 & $14.63 \pm 7.29$ & $55.98 \pm 33.12$ \\
7 & $15.58 \pm 13.51$ & $27.96 \pm 12.38$ \\
Mean & $21.01 \pm 11.67$ & $35.04 \pm 14.72$ \\
\hline
\end{tabular}




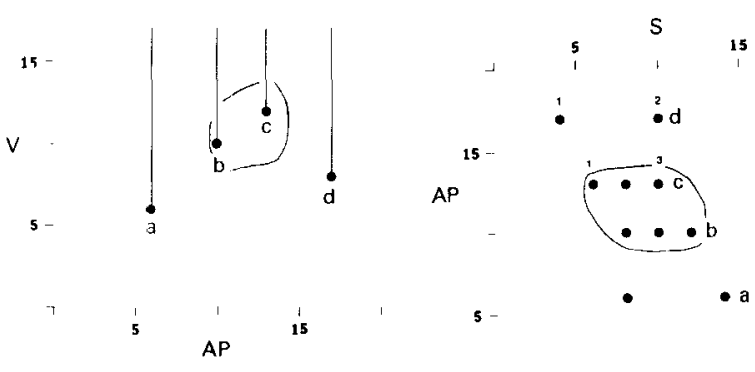

Fig. 3. This shows the stereotactic positions of the tips of 10 electrodes, 6 within the VPL (electrodes $b_{1}, b_{2}, b_{3}, c_{1}, c_{2}$ and $\left.c_{3}\right)$ and 4 around the VPL $\left(a_{1}, a_{2}, d_{1}\right.$ and $\left.d_{2}\right)$, in another animal. The left half of the diagram shows the array as if viewed from the right side; the right half of the diagram gives the view from above. Stereotactic axes are indicated as AP, S and $\mathrm{V}$ with coordinates in $\mathrm{mm}$, and the outline of the VPL is sketched in.

\section{TABLE III}

Amplitude of the positivity recorded at each of the 10 sites shown in Fig. 3 after thalamic ischaemia.

\begin{tabular}{lc}
\hline Electrode & Amplitude $(\mu \mathrm{V})$ \\
\hline a1 & 9.5 \\
a2 & 8.6 \\
b1 & 12.7 \\
b2 & 16.9 \\
b3 & 23.1 \\
c1 & 40.9 \\
c2 & 100.7 \\
c3 & 66.3 \\
d1 & -7.7 \\
d2 & 4.7 \\
\hline
\end{tabular}

between the potentials recorded from different electrodes, with electrodes $\mathrm{C} 2$ and $\mathrm{C} 3$ showing the greatest amplitude. In a previous series (Ladds et al. 1984), we recorded directly from the region of the medial lemniscus during electrode implantation (Fig. 4); the large positive potential seen was mainly confined to the lemniscal tract. A comparison of this potential with the ischaemic responses recorded in the present series from the thalamus (Fig. 2) shows the latencies and wave shapes to be essentially the same.

\section{Discussion}

From the above results it is clear that the potentials recorded within the thalamus are complex and involve several generators. Following the production of thalamic ischaemia, the cortical potentials $\mathrm{N} 10$ and P15 (equivalent to N20 and $\mathrm{P} 25 / 30$ in the human) were abolished because of loss of thalamo-cortical transmission; however, there was a residual cortical positive potential corresponding in latency and morphology to the remaining positive wave recorded in the thalamus. We have seen previously (Ladds et al. 1984) that in the monkey, with occlusion of the middle cerebral artery, the loss of the cortical waves N10 and P15 does not produce changes in the thalamic response (Fig. 5). Therefore, changes in the VPL potential seen in the present series are probably not due to loss of feedback from the cortex, and the enhancement of the VPL response after local dense ischaemia indicates that this positivity is generated outside the thalamus.

Under these assumptions, the difference between the control and final responses at any one VPL recording site will be an estimate of the response generated locally. An example of such digital subtraction is shown in Fig. 6; the result is a negative wave. The VPL structure is complex (Scheibel and Scheibel 1966) and could include closed field neuronal configurations (Llinás and Nicholson 1974) within which an active electrode would show a negative potential, with respect to a distant reference, following an afferent volley from the medial lemniscus. If the afferent volley consisted of a single synchronised impulse, such a negative wave would then be expected to have a relative latency consistent with post-synaptic activity. The nearly synchronous onset of the positive and negative waves in Fig. 6 might appear at first sight to contradict this explanation. However, the afferent volley entering the VPL following peripheral nerve stimulation actually contains not one component but several components mutually dispersed in time, giving rise to a fairly broad ( 5 msec) wave, while in Fig. 6 the onset of the positive wave appears more abrupt than that of the negative wave. The possibility that the negative wave is, in fact, real and originates within 


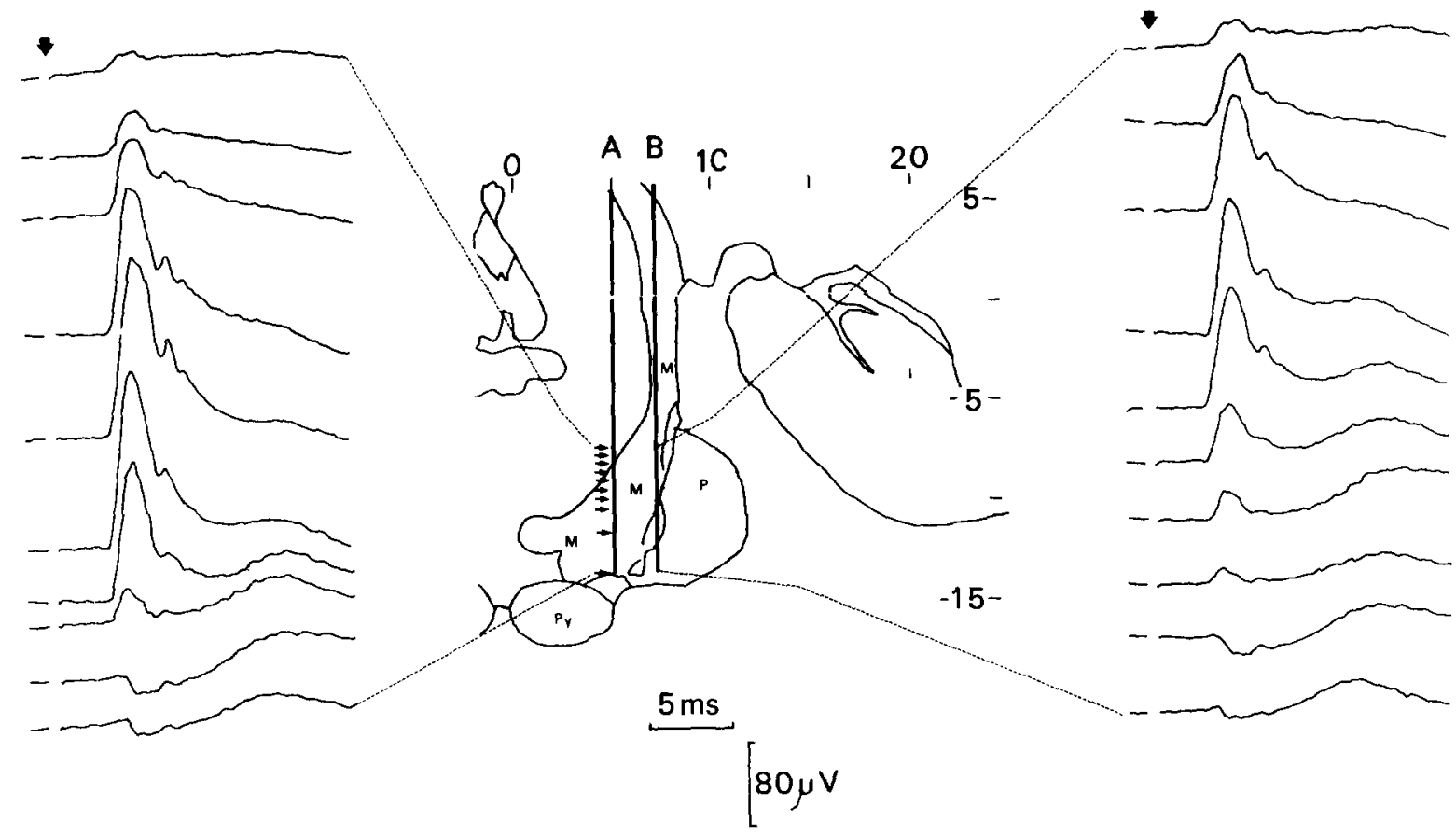

Fig. 4. Responses recorded from 2 electrodes (A and B) during stereotactic implantation into the medial lemniscus. The sites of recording of each response are indicated by the arrows on the electrode tracks. $\mathbf{M}=$ medial lemniscus; $P=$ peduncularis cerebellaris medius; $\mathrm{Py}=$ tractus pyramidalis; $\mathrm{H}=$ hippocampus. Reproduced by permission (as Fig. 1 ).

closed-field thalamic neuronal arrays is therefore not precluded, and we think this is one of the likely factors explaining our data. Anderson et al. (1964) recorded a brief initial negative wave in the thalamus following somatosensory stimulation. They also observed spike discharges superimposed on the negative wave, and our observation that the oscillatory wavelet activity is strictly confined to the region of the VPL (Fig. 1) is consistent with this.

$$
\text { A }
$$
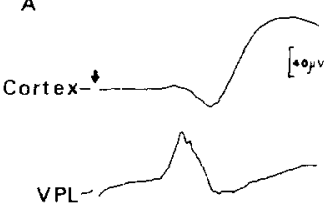
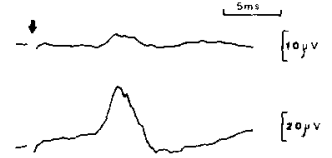

Fig. 5. SEPs recorded in a previous study (Ladds et al. 1984) from the cortical surface and in the VPL before (A) and after (B) occlusion of the ipsilateral middle cerebral artery in the baboon, illustrating the residual surface positivity and the preservation of the VPL response.

Local

Flow

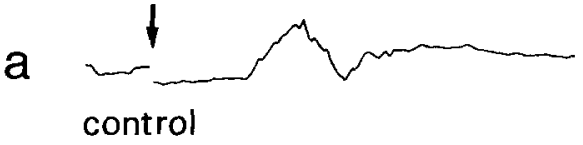

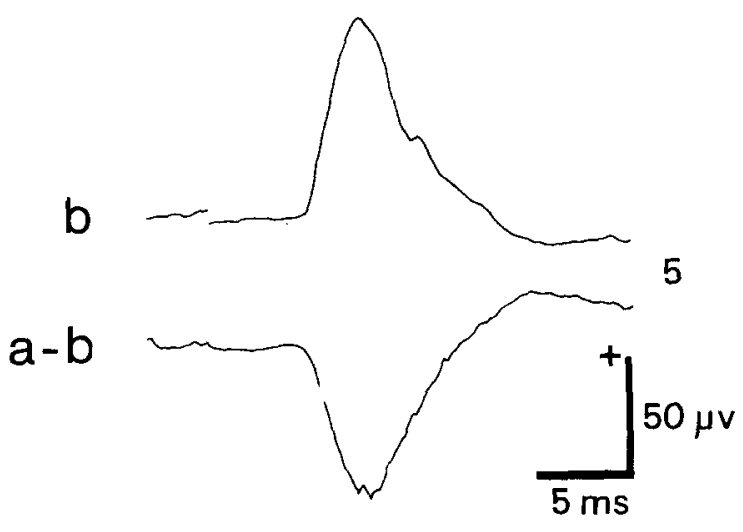

Fig. 6. Responses from one electrode in the VPL during the control phase (a) and after ischaemia (b), and the subtraction (performed by computer) of b from a. Local blood flow (in $\mathrm{ml} / 100 \mathrm{~g} / \mathrm{min}$ ) associated with each response is indicated. 
In the normally perfused VPL, then, the response to median nerve stimulation appears to be the sum of 3 components: a positive component originating outside the VPL, the small oscillatory wavelets originating locally, and a negative potential resulting from the VPL acting as a sink. The first of these components may be traced up to the scalp with diminishing amplitude but unchanged latency; it most likely originates in the dipole complex associated with the approach of the afferent volley in the medial lemniscus, conduction in which is unaffected by VPL ischaemia. However, the marked variation in the amplitude of this component with position within the VPL, as indicated by Fig. 3 and Table III for electrode sets $b$ and $c$, together with the similarity of the wave forms to 'killed end' potentials, suggests that the positivity also includes contributions originating within the VPL itself, from terminal depolarisations in the afferent fibres. When the thalamus is made ischaemic, the locally generated wavelets and the thalamic negativity will be abolished because they are synaptically based, but the first component will remain, appearing as a positive enhancement of the original response.

As we observed (Fig. 4), this enhancement will not be seen outside the VPL. The large variation in the degree of enhancement with the position of the recording electrode (Fig. 2) is explainable on this hypothesis as a corresponding variation with position of the relative proportions of the 3 superimposed components (Fig. 7).

In considering the origin of the electrophysiological changes recorded within the VPL, the question arises whether the production of a focal ischaemic lesion could have distorted the volume conductor properties of the tissue so as to enhance the positive response, either via the reduction in local blood volume (an effect which would be immediate and progressive with successive steps of ischaemia), or through the generation of intracellular interstitial oedema. No such progressive electrophysiological effect was observed prior to the major changes described, so that decreased blood volume is unlikely to have been a significant factor in our results. In addition, although from earlier studies of the production of ischaemic oedema in the cerebral cortex (e.g., Branston et al.
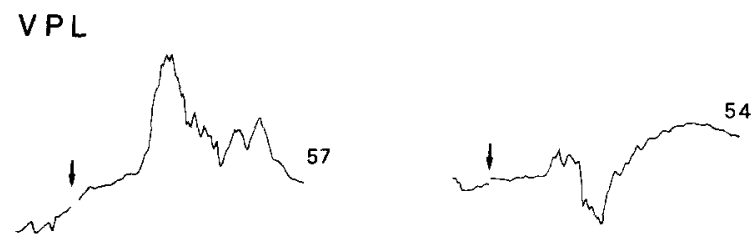

a

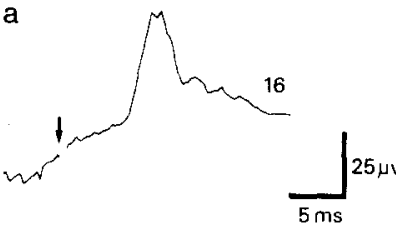

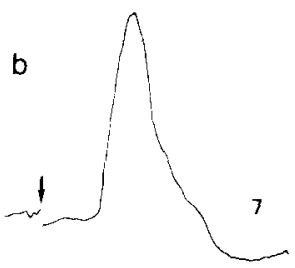

Fig. 7. Responses from 2 electrodes ( $\mathrm{a}$ and $\mathrm{b}$ ) in the thalamus before thalamic ischaemia (upper responses) and after ischaemia (lower responses). The amplitude of the response in a changes very little after ischaemia, the positive component (see Discussion) being predominant. In contrast, when this component is relatively well balanced by the local potentials before ischaemia, as seen in $b$, there is a large increase in amplitude after ischaemia. Local blood flow is indicated numerically as before.

1980) we may infer that some degree of localised oedema would have been generated under the present experimental protocol, any changes of the interstitial or intracellular volumes would have been minor, and it is difficult to see how these could have altered the volume conductor properties so as to produce enhancements of the VPL response as were observed with such consistent polarity.

This study was funded by the Medical Research Council.

We are grateful to Miss $\mathrm{K}$. Cunningham and $\mathrm{Mr}$. C. Bashford for their technical support and to Miss J. Crisell for secretarial assistance.

\section{References}

Abbruzzese, M., Favale, E., Leandri, M. and Ratto, S. New subcortical components of the cerebral somatosensory evoked potential in man. Acta neurol. scand., 1978, 57: 325-332.

Allison, T. and Hume, A.L. A comparative analysis of shortlatency somatosensory evoked potentials in man, monkey, cat and rat. Exp. Neurol., 1981, 72: 592-611.

Anderson, P., Brooks, C.McC., Eccles, J.C. and Sears, T.A. The ventro-basal nucleus of the thalamus: potential fields, syn- 
aptic transmission and excitability of both presynaptic and postsynaptic components. J. Physiol. (Lond.), 1964, 174: 348-369.

Branston, N.M., Bell, B.A., Hunstock, A. and Symon, L. Time and flow as factors in the formation of post-ischaemic edema in primate cortex. Adv. Neurol., 1980, 28: 291-298.

Branston, N.M., Ladds, S., Symon, L. and Wang, A.D. Comparison of the effects of ischaemia on early components of the somatosensory evoked potential in brainstem, thalamus and cerebral cortex. J. Cereb. Blood Flow Metab., 1984, 4: 68-81.

Broughton, R. In: E. Donchin and D.B. Lindsley (Eds.), Averaged Evoked Potentials: Methods, Results and Evaluations. NASA, Washington, DC, 1969: 79-84.

Cracco, R.Q. The initial positive potential of the human scalprecorded somatosensory evoked response. Electroenceph. clin. Neurophysiol., 1972, 32: 623-629.

Cracco, R.Q. and Cracco, J.B. Somatosensory evoked potential in man: far-field potentials. Electroenceph. clin. Neurophysiol., 1976, 41: 460-466.

Crespi, V., Mandelli, A. and Minoli, G. Short-latency somatosensory evoked potentials in patients with acute focal vascular lesions of the supratentorial somesthetic pathways. Acta neurol. scand., 1982, 65: 274-279.

Desmedt, J.E. Non-invasive analysis of the spinal cord generators activated by somatosensory input in man: near field and far field potentials. Exp. Brain Res., 1984, Suppl. 9: 45-62.

Hammond, E.J., Wilder, B.J. and Ballinger, W.E. Electrophysiologic recordings in a patient with a discrete unilateral thalamic infarction. J. Neurol. Neurosurg. Psychiat., 1982, 45: $640-643$

Jones, S.J. Short-latency potentials recorded from the neck and scalp following median nerve stimulation in man. Electroenceph. clin. Neurophysiol., 1977, 43: 853-863.

Ladds, A., Branston, N.M., Wang, A. and Symon, L. Correlation of the somatosensory evoked potential with changes in local tissue blood flow in brainstem, thalamus and cerebral cortex during progressive ischaemia in primates. In: R.H. Nodar and C. Barber (Eds.), Evoked Potentials, II. Butterworth, Boston, MA, 1984: 497-505

Llinás, R. and Nicholson, C. Analysis of field potentials in the central nervous system. In: A. Rémond (Ed.), Handbook of Electroenceph. clin. Neurophysiol., Vol. 2B. Elsevier, Amsterdam, 1974: 63-83.

Nakanishi, T., Shimada, Y., Sakuta, M. and Toyokura, Y. The initial positive component of the scalp-recorded somatosensory evoked potential in normal subjects and in patients with neurological disorders. Electroenceph. clin. Neurophysiol., 1978, 45: 26-34.

Nakanishi, T., Tamaki, M., Ozaki, Y. and Arasaki, K. Origins of short-latency somatosensory evoked potentials to median nerve stimulation. Electroenceph. clin. Neurophysiol., 1983, 56: 74-85.

Noël, P. and Desmedt, J.E. Somatosensory cerebral evoked potentials after vascular lesions of the brainstem and diencephalon. Brain, 1975, 98: 113-128.

Rosner, B.S., Goff, W.R. and Allison, T. Properties of cerebral somatic evoked responses in unanesthetised cebus monkey. Electroenceph. clin. Neurophysiol., 1963, Suppl. 24: 43-54.

Scheibel, M.E. and Scheibel, A.B. Patterns of organisations in specific and non-specific thalamic fields. In: D.P. Purpura and M.D. Yahr (Eds.), The Thalamus. Columbia University Press, New York, 1966: 13-46.

Stöhr, M., Dichgans, J., Voigt, K. and Buettner, N.W. The significance of somatosensory evoked potentials for localisation of unilateral lesions within the cerebral hemispheres. J. neurol. Sci., 1983, 61: 49-63.

Suzuki, I. and Mayanagi, Y. Intracranial recording of short latency somatosensory evoked potentials in man: identification of origin of each component. Electroenceph. clin. Neurophysiol., 1984, 59: 286-296.

Vajda, J., Branston, N.M., Ladds, A. and Symon, L. A model of selective experimental ischaemia in the primate thalamus. Stroke, 1985, 16: 494-501. 